\title{
Science opportunities with the Space Infrared Telescope Facility (SIRTF)
}

\author{
Michelle Thaller \\ SIRTF Science Center, IPAC, CalTech, Pasadena, CA 91125, USA
}

\section{SIRTF}

After a quarter century of dreams and designs, the Space Infrared Telescope Facility (SIRTF) has begun formal development, with a planned launch date of December 2001. SIRTF consists of a $85 \mathrm{~cm}$ telescope and three cryogenicallycooled science instruments capable of performing imaging, photometry, and spectroscopy in the 3-180 $\mu \mathrm{m}$ wavelength range. Incorporating the latest in largeformat infrared detector arrays, SIRTF offers orders-of-magnitude improvements in capability over existing programs. One of the many innovative aspects of this observatory is the Legacy Science program, in which hundreds, perhaps thousands of hours of observing time will be allocated to coherent groups of scientists to carry out programs whose scientific data, upon archiving, are of general and lasting importance to the broad community. The capabilities of this telesope, and the possibiltiy of participating in a Legacy Science proposal, offer the community of hot stellar astronomers an unprecedented scientific opportunity. 